\title{
Prospective, observational, multicenter study on minimally invasive gastrectomy for gastric cancer: robotic, laparoscopic and open surgery compared on operative and follow-up outcomes - IMIGASTRIC II study protocol
}

Jacopo Desiderio ${ }^{1,2^{*}}$, Jian-Xian Lin $^{3}$, Enrique Norero ${ }^{4}$, Felice Borghi ${ }^{5}$, Alessandra Marano ${ }^{5}$, Fabio Cianchi ${ }^{6}$, Benedetta Badii ${ }^{6}$, Feng Q $i^{7}$, Tong Liu ${ }^{7}$, Daniel Reim ${ }^{8}$, Alexander Novotny ${ }^{8}$, Andrea Coratti ${ }^{9}$, Francesco Guerra ${ }^{9}$, Ninh T Nguyen ${ }^{10}$, Orhan Alimoglu ${ }^{11}$, Ibrahim Ali Ozemir ${ }^{11}$, Steven Brower ${ }^{12}$, Francesca Bazzocchi ${ }^{13}$, Andrea Avanzolini ${ }^{14}$, Leonardo Solaini ${ }^{14}$, Orestis Ioannidis ${ }^{15}$, Christos Chatzakis ${ }^{15}$, Lydia Loutzidou ${ }^{15}$, Arda Isik ${ }^{16}$, Maurizio Cesari ${ }^{17}$, Graziano Pernazza ${ }^{18}$, Juan-Santiago Azagra ${ }^{19}$, Martine Goergen ${ }^{19}$, Yanbing $Z_{\text {Zhou }}^{20}$, Shougen Cao ${ }^{20}$, Johan Gagnière ${ }^{21}$, Denis Pezet ${ }^{21}$, Simone D'Imporzano ${ }^{22}$, Olivier Saint-Marc ${ }^{23}$, Rémy Sindayigaya ${ }^{23}$, Lu Zang ${ }^{24}$, Natalie G. Coburn ${ }^{25}$, Cuneyt Kayaalp ${ }^{26}$, Giacomo Arcuri ${ }^{1}$, Francesco Giovanardi ${ }^{1}$, Stefano Trastulli ${ }^{1}$, Chao-Hui Zheng ${ }^{3}$, Amilcare Parisi ${ }^{1}$, Chang-Ming Huang ${ }^{3}$

${ }^{1}$ St. Mary's Hospital, Department of Digestive Surgery, Terni, Italy.

${ }^{2}$ La Sapienza University of Rome, Department of Surgical Sciences - PhD program in advanced surgical technologies, Rome, Italy.

${ }^{3}$ Department of Gastric Surgery; Department of General Surgery; Key Laboratory of Ministry of Education of Gastrointestinal Cancer, Fujian Medical University Union Hospital, Fuzhou, Fujian Province, PR China.

${ }^{4}$ Hospital Dr. Sotero del Rio, Esophagogastric Surgery Unit, Digestive Surgery Department, Pontificia Universidad Catolica de Chile, Chile.

${ }^{5}$ Department of Surgery, General and Oncologic Surgery Unit, Santa Croce e Carle Hospital, Cuneo, Italy.

${ }^{6}$ Center for Oncological Minimally Invasive Surgery (COMIS), Department of Experimental and Clinical Medicine, University of Florence, Florence, Italy.

${ }^{7}$ Department of General Surgery, Tianjin Medical University General Hospital, Tianjin, PR China.

${ }^{8}$ Department of Surgery, Klinikum rechts der Isar, School of Medicine, Technical University of Munich (TUM), Munich, Germany.

${ }^{9}$ Division of Oncological and Robotic General Surgery, Careggi University Hospital, Florence, Italy

${ }^{10}$ Department of Surgery, University of California Irvine Medical Center, Orange, CA, USA

${ }^{11}$ Department of General Surgery, Istanbul Medeniyet University, School of Medicine, Istanbul, Turkey.

${ }^{12}$ The Lefcourt Family Cancer Treatment and Wellness Center and Departments of Surgical Oncology and Hepatobiliary Surgery, Englewood Hospital and Medical

Center, Englewood NJ, USA.

${ }^{13}$ Department of Abdominal Surgery, “Casa Sollievo della Sofferenza” Hospital, San Giovanni Rotondo, Italy.

${ }^{14}$ General and Oncologic Surgery, Morgagni-Pierantoni Hospital, Ausl Romagna, Forli, Italy.

${ }^{15}$ Fourth Surgical Department, Medical School, Aristotle University of Thessaloniki, Thessaloniki, Greece.

${ }^{16}$ Division of General Surgery, School of Medicine, Erzincan Binali Yildirim University, Erzincan, Turkey.

${ }^{17}$ Department of General Surgery, Hospital of Città di Castello, USL1 Umbria. Città di Castello, Italy.

${ }^{18}$ Division of Robotic General Surgery, San Giovanni Addolorata Hospital, Rome, Italy.

${ }^{19}$ Department of General and Minimally Invasive Surgery, Centre Hospitalier de Luxembourg, Luxembourg City, Luxembourg.

${ }^{20}$ Department of Gastrointestinal Surgery, Affiliated Hospital of Qingdao University, Qingdao City, Shandong Province, PR China.

${ }^{21}$ Department of Digestive and Hepatobiliary Surgery, Hôpital Estaing, CHU Clermont-Ferrand, Clermont-Ferrand, France.

${ }^{22}$ Esophageal Surgery Unit, Tuscany Regional Referral Center for the Diagnosis and Treatment of Esophageal Disease, Medical University of Pisa. Pisa, Italy.

${ }^{23}$ Service de Chirurgie Digestive, Endocrinienne et Thoracique, Centre Hospitalier Régional, Orleans, France.

${ }^{24}$ Department of Surgery, Ruijin Hospital, Shanghai Jiaotong University, School of Medicine. Shanghai, PR China.

${ }^{25}$ Division of General Surgery, Department of Surgery, University of Toronto, Toronto, Ontario, Canada; Institute of Health Policy, Management and Evaluation, University of Toronto, Toronto, Ontario, Canada; Evaluative Clinical Sciences, Sunnybrook Research Institute, Toronto, Ontario, Canada; ICES, Toronto, Ontario, Canada.

${ }^{26}$ Department of General Surgery, İnönü University Faculty of Medicine, Malatya, Turkey.

To Cite

Desiderio J, Lin J-X, Norero E, Borghi $\mathrm{F}$, Marano A, Cianchi F, Badii B, Qi F, Liu T, Reim D, Novotny A, Coratti A, Guerra F, Nguyen NT, Alimoglu O, Ozemir IA, Brower S, Bazzocchi F, Avanzolini A, Solaini L, Ioannidis O, Chatzakis C, Loutzidou L, Isik A, Cesari M, Pernazza G, Azagra J-S, Goergen M, Zhou Y, Cao S, Gagnière J, Pezet D, D'Imporzano S, SaintMarc O, Sindayigaya R, Zang L, Coburn

\section{ABSTRACT}

Background:

Several meta-analyses have tried to define the role of minimally invasive approaches. However, further evidence to get a wider spread of these methods is necessary. Current studies describe minimally invasive surgery as a possible alternative to open surgery but deserving further clarification. However, despite the increasing interest, the difficulty of planning prospective studies of adequate size accounts for the low level of evidence, which is mostly based on retrospective experiences.

A multi-institutional prospective study allows the collection of an impressive amount 
NG, Kayaalp C, Arcuri G, Giovanardi F, Trastulli S, Zheng C-H, Parisi A, Huang C-M. Prospective, observational, multicenter study on minimally invasive gastrectomy for gastric cancer: robotic, laparoscopic and open surgery compared on operative and follow-up outcomes - IMIGASTRIC II study protocol. J Gastric Surg 2019; 1(1): 9-15

\section{Pubblication history}

Received: October 25, 2019

Revised: November 20, 2019

Accepted: December 5, 2019

Article in press: December 10, 2019

Published online: December 16, 2019

\section{Correspondence to}

Dr. Jacopo Desiderio

Department of Digestive Surgery,

St Mary's Hospital, Terni 05100, Italy

j.desiderio@aospterni.it

Telephon: +393425595828 of data to investigate various aspects of minimally invasive procedures with the opportunity of developing several subgroup analyses.

A prospective data collection with high methodological quality on minimally invasive and open gastrectomies can clarify the role of different procedures with the aim to develop specific guidelines.

Methods and analysis:

a multi-institutional prospective database will be established including information on surgical, clinical and oncological features of patients treated for gastric cancer with robotic, laparoscopic or open approaches and subsequent follow-up.

The study has been shared by the members of the International study group on Minimally Invasive surgery for GASTRIc Cancer (IMIGASTRIC)

The database is designed to be an international electronic submission system and a HIPPA protected real time data repository from high volume gastric cancer centers.

\section{Ethics:}

This study is conducted in compliance with ethical principles originating from the Helsinki Declaration, within the guidelines of Good Clinical Practice and relevant laws/regulations.

\section{Trial registration number:}

NCT02751086 


\section{Background}

Oncologic gastric surgery represents a major field of interest and development for minimally invasive surgery. Several institutions have continuously published reports regarding their experiences and progress, which has allowed authors of systematic reviews and metaanalysis to try to define the role of minimally invasive surgery (laparoscopy, robotic) by comparing it with the open approach[1-3].

However, the current level of evidence has not yet allowed for defining and sharing rules for the application of minimally invasive approaches in common surgical practice.

Guidelines describe laparoscopy as a possible alternative to open surgery for early gastric cancer[4]; meanwhile, robotic surgery possesses intrinsic technological advantages. However, researchers have not yet verified these advantages through studies with an appropriate level of evidence[5]. Research in this field aims to assess the effects on perioperative outcomes and the patient's quality of life while still respecting oncological principles. The increasing attention that researchers have paid to these approaches are unfortunately limited by the incomplete data currently available[5].

Robotic systems have revolutionized the way we perform minimally invasive surgery and have facilitated the evolution of traditional laparoscopy. Surgeons can overcome the limits of traditional laparoscopy through three-dimensional vision, articulated instruments, and the absence of tremors, thus creating greater dexterity and precision in dissection and suturing movements. These are key elements when performing an extended lymphadenectomy for gastric cancer, and complex and gentle reconstruction to restore digestive continuity.

Despite the technological advances, several issues are currently subject to debate. The most important issue is ensuring proper oncological surgery by performing an adequate lymphadenectomy with minimally invasive approaches. Researchers still regard nodal clearance as an important factor influencing long-term survival[6-13]. In randomized trials[3], laparoscopy demonstrated the removal of at least 15 lymph nodes, as required by international guidelines[14]; however, a significant difference in favor of open surgery resulted in the total number of lymph nodes harvested.

Robotic surgery can facilitate better D2 dissection. This advanced technology clearly possesses intrinsic advantages for this surgical step, but researchers have not yet proven and verified them through appropriate trials: only four studies[15-18] have compared robotic surgery with the open approach, and only one study shows a statistically significant difference versus laparoscopy[19].

Among the intraoperative outcomes, most of the available studies found that blood loss was in favor of minimally invasive surgery. This finding has achieved high statistical significance for laparoscopy in Vinuela's meta-analysis of RCTs[3]. Meanwhile, with regard to robotic surgery, a general consensus among different studies seems to have detected some advantages over laparoscopy and open surgery in reducing operative bleeding[19, 20]. However, several studies have also reported conflicting results[21, 22].

Regarding the post-operative period, the largest RCT[23], which was performed by the Korean Laparoscopic Gastrointestinal Surgery Study Group, found no significant difference between laparoscopy and open surgery in overall complications. Other studies[3] have shown a significant reduction in medical and minor surgical complications when using laparoscopy. Researchers have obtained inconsistent findings in studies on robotic surgery in terms of demonstrating differences compared to laparoscopy in the analysis of complications[22, 24, 25].

Overall, minimally invasive surgery has demonstrated relevant advantages over open surgery with regard to postoperative hospital stays[1, 26-28], despite the extreme heterogeneity among studies. Some evidence[ $[18$, 29] has indicated that patients who underwent robotic gastrectomy could be discharged at an earlier date than patients who underwent open or laparoscopic gastrectomy. However, the low number of studies in this field and the high heterogeneity weaken this conclusion. Manually handling organs during surgery is an important contributor to the inflammatory response after surgery[27, 28, 30]. Theoretically, smaller robotic instruments may cause less inflammation than the instruments used in other approaches. Therefore, postoperative bowel recovery in the robotic group may occur sooner, but this hypothesis still must be proven.

New research must contribute to the current literature in order to define the role of different surgical approaches, and researchers still have to explore many aspects of minimally invasive surgery for gastric cancer[5, 31, 32]. We have a long way to go. Currently, the scientific community is wondering what strategies should be adopted in future studies.

\section{Rationale}

A review of the scientific literature[5], which was recently published by the IMIGASTRIC study group, aimed to perform a more complete analysis of the current situation regarding performing minimally invasive surgery for gastric cancer. Significant limitations were found in the analyzed studies, including:

- Small samples of patients, mostly low-quality comparative studies

- Selection bias in the comparison groups (e.g. stage, extent of lymphadenectomy)

- Absence of subgroup analysis in significant research fields

- Lack of information on the surgical techniques adopted A large prospective multicenter registry could thus be the optimal way to clarify the role of minimally invasive surgery for gastric cancer and permit the evaluation of its short and long-term effects. A working basis for analyzing outcomes of interest and obtaining directions for guidelines and future study developments can also be created. The following would be the main advantages of a large prospective multicenter registry:

-Achieving a large sample of patients

-Collecting multiple variables, allowing for the making of a comprehensive statistical report

-Standardizing the methodology to be adopted, thus 
increasing accuracy

-Bringing together the experiences of both East and West to discover shared points

A prospective registry can become a powerful tool that can guide research in this field to new developments and pave the way for other investigational opportunities.

\section{Preliminary data}

A research group was first established in 2014 and after sharing a specific study protocol, data collection officially started at the end of 2015 through a retrospective chart review (IMIGASTRIC) [5, 32, 33].

More than 4200 cases have been entered in the registry to date.

$60 \%$ of entered cases are from Asia, but over 1600 cases were collected by western institutions. About 2600 patients underwent a MIS approach.

A first comparison including a matching analysis regarding operative results, postoperative recovery, and complications was published[34].

The operative time was significantly longer in the Robotic group than the other two groups. A statistical difference in favor of the open group was also observed when compared with the laparoscopic group. A reduction in blood loss resulted in favor of both minimally invasive approaches vs the open group and particularly slightly in favor of the laparoscopic group when compared with the robotic group. Regarding the number of retrieved lymph nodes, MIS confirmed to guarantee an adequate number of nodes for pathological assessment with no significant difference with the open approach. No differences were found regarding intraoperative complications or the residual tumor status. The conversion rate was $5.3 \%$ in the LG and $4.6 \%$ in the RG with no significant difference. A significantly shorter hospital stay was found in both MIS group vs the OG, without differences between the LG and RG.

All steps in the patients' recovery status happened faster in both minimally invasive approaches than the open surgery. A small benefit was found to be significant in the minimally invasive groups vs the OG in intravenous analgesic discontinuation. The other outcomes showed a slight advantage in favor of minimally invasive surgery. A decrease in the number of patients experiencing postoperative complications was shown in both the robotic and laparoscopic group versus the open group, however this difference was not statistically significant. No differences were shown in surgical and nonsurgical complications, as well as regarding the type of complication. Leakage, bleeding, and pancreatic fistula were the most observed surgical complications. Pneumonia and urinary were the most common medical complications. Majority of cases were low grade complications based on the Clavien Dindo classification (76.9\%, overall). The number of patients requiring reoperation did not differ among the three groups.

Anastomotic leakage was the most relevant surgical complication. Therefore, further analysis is reported. No differences in the overall leakage rate was shown between groups, as well as the distribution by different sites. The leak-related reoperation rate did not differ among the three groups.

Laparoscopic and robotic surgery, in this report, showed safety in ensuring oncological radicality with short hospitalization, benefits in all patients' functional recovery steps and a lower trend in complications rates.

\section{Methods and analysis}

\section{General study design:}

The overall purpose is to develop and maintain a multi-institutional database comprising of information regarding surgical, clinical and oncological features of patients that will be treated for gastric cancer with robotic, laparoscopic or open approaches and subsequent follow-up.

The main objective is to compare the three surgical arms on surgical and clinical outcomes, as well as on the oncological follow-up.

\section{Specific aims:}

AIM 1: To compare robotic and laparoscopic surgery to the open approach in terms of safety and feasibility based on the intraoperative outcomes.

AIM 2: To verify the respect of oncological principles through minimally invasive approaches by comparing histopathological findings to open surgery.

AIM 3: To compare the three treatment arms regarding the postoperative course.

AIM 4: To compare the incidence, types and severity of inhospital and long-term complications after gastrectomy by the three approaches according to the Clavien-Dindo classification system[35]

AIM 5: To verify whether minimally invasive approaches ensure the same effectiveness as open surgery in terms of overall survival and disease-free survival at the scheduled endpoints.

\section{Eligibility}

Every patient is required to meet all the inclusion criteria and none of the exclusion criteria.

Inclusion criteria

- Histologically proven gastric cancer

- Preoperative staging work-up performed by upper endoscopy and/or endoscopic ultrasound, and CT scan and in accordance to international guidelines[14]

- Early Gastric Cancer

- Advanced Gastric Cancer

- Patients treated with curative intent in accordance to international guidelines[14]

Exclusion criteria

- Distant metastases: peritoneal carcinomatosis, liver metastases, distant lymph node metastases, Krukenberg tumors, involvement of other organs

- Patients with high operative risk as defined by the American Society of Anesthesiologists (ASA) score $>4$

- History of previous abdominal surgery for gastric cancer

- Synchronous malignancy in other organs

- Palliative surgery 
Data collection (main variables)

Patient Demographics

- Year of birth

- Sex

- BMI

- Surgical risk (ASA score)

- Concomitant illness

- Previous surgery

- Staging laparoscopy

- Neoadjuvant chemo/radio-therapy

- Pre-operative blood samples

Surgery

- Operation date

- Type of surgical approach (Open, Laparoscopy, Robotic)

- Type of gastric resection (Total gastrectomy, Distal gastrectomy, Pylorus-preserving gastrectomy, Proximal gastrectomy)

- Type of recostruction

- Anastomosis approach (intracorporeal, extracorporeal)

- Anastomosis performance (linear stapler, circular stapler, hand-sewn)

- Site and lenght of mini-laparotomy

- Placement of intra abdominal drain

- Placement of nasogastric tube

- Total operative time

- Estimated blood loss

- Conversion to open surgery

- Intraoperative complications

- Extent of lymphadenectomy

- Resection margins

- Surgical radicality (R)

- Number of retrieved lymph nodes

Tumor

- Location and diameter

- Depth of invasion (T classification)

- Number of metastatic lymph nodes

- Lymph node status (N classification)

- AJCC pathological stage

- Histological type

Post-operative clinical findings

- ERAS protocols adopted

- Length of postoperative hospital stays

- Patient mobilization (POD no.)

- Liquid diet (POD no.)

- Soft solid diet (POD no.)

- First flatus (POD no.)

In-hospital post-operative complications

- Type of complication

- Reoperation for complication

- Clavien - Dindo grade[35]

Early and late complications after discharge

- Date of occurrence

- Type of complication

- Death related to the complication

- Need of surgery

Follow-up

- Adjuvant chemo/radio-therapy

- Date of follow up visits

- Patient status at follow-up visits (alive, dead, lost to follow-up assessment)

- Disease-free or not during follow-up

\section{Primary outcome measures}

- Safety and feasibility of procedures: rate of intraoperative complications, rate of intraoperative death, rate of conversion to open surgery, average of estimated blood loss during surgery.

- Respect of oncological principles: average of retrieved lymph nodes, rate of patients achieving $\mathrm{R} 0$ resection, rate of patients achieving specimen margins free of disease, at the histopathological analysis.

- Effectiveness of surgery: overall survival and disease-free survival achieved at 1, 2, 3, 4, 5 years from surgery.

\section{Secondary outcome measures}

- Recovery after surgery: rate of post-operative blood transfusion, average of time to patient mobilization, average of time to resumption of peristalsis, average of time to starting oral intake, average of time to first flatus, average of length of intravenous antibiotic use, average of length of intravenous analgesic use, average of hospitalization after surgery until discharge.

- Early and long-term complications after surgery: rate of total complications, rate of specific surgical complications, severity of complications scored on the Clavien-Dindo classification system[35].

\section{Statistical analysis}

Based on the data of the registry every investigator can perform all the statistical analysis he needs for his researchers' purposes, while a basic analysis for monitoring the study will be performed as follows. SPSS version 23 will be used to carry out the interim data analyses.

The dichotomous variables will be expressed as numbers and percentages, while continuous variables as mean and SD, or median and IQR (minimum and maximum values). Continuous variables will be compared using one-way ANOVA analysis of variance with post hoc multiple comparison by Tukey's procedure.

Pearson's $\chi^{2}$ test or Fisher's exact test, as appropriate, will be used for analysis of categorical data.

The $\mathrm{Z}$ test with the Bonferroni correction will be used to evaluate statistical significance among the surgical approaches: Robotic, Laparoscopy, Open.

For each of these tests a value of $a<0.05$ will be considered statistically significant.

Subgroup analysis will be done to evaluate the overall survival, by considering patients with follow-up details at the data extraction time.

Overall survival will be computed from the day of surgery to the day of death or to the last reported followup visit. Overall survival analysis will be performed using Kaplan-Meier curves. Comparison between different groups will be carried out using the log rank test.

\section{Sample size}

It is estimated from the IMIGASTRIC I study that the rate of procedures performed with minimally invasive surgery at referral institutes for gastric cancer, considering patients who follow inclusion and exclusion 
criteria of this protocol, is of $65 \%$.

According to the number and volume of the participating centers and to reach a sample of 1000 subjects treated with laparoscopic or robotic surgery, is estimated that data of at least 1500 patients need to be collected.

\section{Study period and sites}

The database will remain open for data collection (including the participation of other interested Institution) for at least 10 years, considering current available funds from the promoting institution (St. Mary's Hospital of Terni).

It is estimated that the sample size will be reached in 5 years. The maximum length of each patient for the oncological follow-up is 5 years. Subsequent amendments will extend the follow-up period based on the progress of the project.

The study has been shared by the members of the International study group on Minimally Invasive surgery for GASTRIc Cancer (IMIGASTRIC)[32]. The group involves some of the most important researchers and institutes around the world for the treatment of gastric cancer and began working in 2014.

\section{Ethics and dissemination}

\section{Ethical aspects}

All Investigators agree the study is conducted in compliance with ethical principles originating from the Helsinki Declaration, with the guidelines of Good Clinical Practice (GCP) and with applicable laws.

Investigators shall undertake to act according to the rules of the Institutional Review Board (IRB) and Ethics Committee (EC) regarding the prospective collection of data.

\section{Potential risks and safety management}

Participation in the research registry involves the potential risks of a breach of

confidentiality of the medical record information and associated privacy of the participants.

Confidentiality and data security will be ensured by:

1) removing direct participant identifiers;

2) limiting access to information using the assignment of codes;

3) limiting access to information to center investigators.

Risks are minimized using appropriate tailor-made systems.

Data will be collected and recorded by all institutions through a specific online software (https://imigastric. logix-software.it/).

The system provides the following safeguards:

1. Data entry of patient information complies with the most stringent privacy regulations; sensitive data are not recorded on a server, but the software generates a reference code.

2. For each patient, the system generates an IT folder containing six specific areas to complete.

3. The software is designed to guide the user in data entry, thus avoiding the generation of errors.

4. The different fields are filled in by selecting the various options from drop-down menus available for each parameter. All characteristics to be entered have been previously standardized, without the need to write anything else when filling in the fields. Any considered variable derives from an analysis of all the data reported in previous studies found in the literature and in accordance with accepted guidelines[14].

5. The software uses predefined control instruments.

The data and safety monitoring plan for the research registry will involve routine monitoring by the organizing committee of any conditions that may negatively impact the confidentiality of information contained within the research registry.

In addition, any unauthorized access to medical record information contained within the research registry or to the database linking the registry information to participant direct identifiers will be reported to a data and safety monitoring board.

\section{Study's website}

A Study's website is available at: www.imigastric.com to obtain information and follow the news of the project. Contact information for the organizing secretariat and the coordinating staff is available there. Interested centers can join this prospective registry.

\section{Publications}

Each participating center, with equal right, will be able to access the data of the registry, perform statistical analysis, discuss the results, and freely write scientific manuscripts. However, each study that is generated based on the registry must be known by all Centers before final publication.

\section{Acknowledgements \\ LOGIX Srl provided IT study solutions.}

\section{Contributors}

$\mathrm{AP}, \mathrm{JD}$ were involved in conception of the study. JXL, EN, FB, AM, FC, BB, FQ, TL, DR, AN, AC, FG, NTN, OA, IAO, SB, FB, AA, LS, OI, CC, LL, AI, MC, GP, JSA, MG, YZ, SC, JG, DP, SDI, OSM, RS, LZ, NGC, CK, GA, ST, $\mathrm{CHZ}, \mathrm{AP}, \mathrm{CMH}$ and JD were involved in designing the study, analyzing the literature, references searching and in drafting the rationale. JXL, ST, CHZ, AP, CMH and JD were involved in description of the study methods.

\section{Funding}

None.

\section{Competing interests}

No benefits in any form have been received or will be received from a commercial party related directly or indirectly to the subject of this article.

\section{Availability of data and materials}

The datasets used and/or analyzed during the current study are available

from the corresponding author on reasonable request.

\section{Ethics approval}

The study was approved by the ethics Committee of CEAS Umbria (Prot. No. 7649/16/ON dated 18/02/2016).

This study is conducted in compliance with ethical 
principles originating from the Helsinki Declaration, within the guidelines of Good Clinical Practice and relevant laws/regulations.

\section{Provenance and peer review \\ Not commissioned; externally peer reviewed.}

\section{Open access}

This is an Open Access article distributed in accordance with the Creative Commons Attribution NonCommercial (CC BY-NC 4.0) license, which permits others to distribute, remix, adapt, build upon this work noncommercially, and license their derivative works on different terms, provided the original work is properly cited and the use is non-commercial. See: http:// creativecommons.org/licenses/by-nc/4.0/

\section{References}

[1] Marano A, Choi YY, Hyung WJ, Kim YM, Kim J, Noh SH. Robotic versus Laparoscopic versus Open Gastrectomy: A Meta-Analysis. Journal of gastric cancer. 2013;13:136-48.

[2] Zong L, Seto Y, Aikou S, Takahashi T. Efficacy evaluation of subtotal and total gastrectomies in robotic surgery for gastric cancer compared with that in open and laparoscopic resections: a meta-analysis. PloS one. 2014;9:e103312.

[3] Vinuela EF, Gonen M, Brennan MF, Coit DG, Strong VE. Laparoscopic versus open distal gastrectomy for gastric cancer: a meta-analysis of randomized controlled trials and high-quality nonrandomized studies. Annals of surgery. 2012;255:446-56.

[4] Japanese Gastric Cancer A. Japanese gastric cancer treatment guidelines 2014 (ver. 4). Gastric Cancer. 2017;20:1-19.

[5] Desiderio J, Jiang ZW, Nguyen NT, Zhang S, Reim D, Alimoglu O, et al. Robotic, laparoscopic and open surgery for gastric cancer compared on surgical, clinical and oncological outcomes: a multi-institutional chart review. A study protocol of the International study group on Minimally Invasive surgery for GASTRIc Cancer-IMIGASTRIC. BM] open. 2015;5:e008198.

[6] Chen QY, Zhong Q, Wang W, Desiderio J, Liu ZY, Xie JW, et al. Development and external validation of a nomogram for predicting the conditional probability of survival after D2 lymphadenectomy for gastric cancer: A multicentre study. Eur J Surg Oncol. 2019;45:1934-42. [7] Desiderio J, Chao J, Melstrom L, Warner S, Tozzi F, Fong Y, et al. The 30-year experience-A meta-analysis of randomised and highquality non-randomised studies of hyperthermic intraperitoneal chemotherapy in the treatment of gastric cancer. Eur J Cancer. 2017;79:1-14.

[8] Huang ZN, Desiderio J, Chen QY, Zheng CH, Li P, Xie JW, et al. Indications for adjuvant chemotherapy in patients with AJCC stage IIa T3N0M0 and T1N2M0 gastric cancer-an east and west multicenter study. BMC Gastroenterol. 2019;19:205.

[9] Lin JX, Lin JP, Desiderio J, Xie JW, Gemini A, Wang JB, et al. Difference in the short-term outcomes of laparoscopic gastrectomy for gastric carcinoma between the east and west: a retrospective study from the IMIGASTRIC trial. J Cancer. 2019;10:4106-13.

[10] Lin JX, Wang ZK, Wang W, Desiderio J, Xie JW, Wang JB, et al. Risk factors of lymph node metastasis or lymphovascular invasion for early gastric cancer: a practical and effective predictive model based on international multicenter data. BMC Cancer. 2019;19:1048,

[11] Lin JX, Yoon C, Desiderio J, Yi BC, Li P, Zheng CH, et al. Development and validation of a staging system for gastric adenocarcinoma after neoadjuvant chemotherapy and gastrectomy with D2 lymphadenectomy. Br J Surg. 2019;106:1187-96.

[12] Wang JB, Zhong Q, Wang W, Desiderio J, Chen S, Liu ZY, et al. Postoperative dynamic survival of gastric cancer patients: A multiinstitutional, international analysis of 22265 patients. J Surg Oncol. 2019;120:685-97.

[13] Zheng ZF, Lu J, Wang W, Desiderio J, Li P, Xie JW, et al. Development and External Validation of a Simplified Nomogram Predicting Individual Survival After R0 Resection for Gastric Cancer: An International, Multicenter Study. Ann Surg Oncol. 2018;25:2383-90. [14] Smyth EC, Verheij M, Allum W, Cunningham D, Cervantes A, Arnold D, et al. Gastric cancer: ESMO Clinical Practice Guidelines for diagnosis, treatment and follow-up. Ann Oncol. 2016;27:v38-v49.

[15] Kim KM, An JY, Kim HI, Cheong JH, Hyung WJ, Noh SH. Major early complications following open, laparoscopic and robotic gastrectomy. The British journal of surgery. 2012;99:1681-7.

[16] Huang KH, Lan YT, Fang WL, Chen JH, Lo SS, Hsieh MC, et al. Initial experience of robotic gastrectomy and comparison with open and laparoscopic gastrectomy for gastric cancer. Journal of gastrointestinal surgery : official journal of the Society for Surgery of the Alimentary Tract. 2012;16:1303-10.

[17] Caruso S, Patriti A, Marrelli D, Ceccarelli G, Ceribelli C, Roviello F, et al. Open vs robot-assisted laparoscopic gastric resection with D2 lymph node dissection for adenocarcinoma: a case-control study. The international journal of medical robotics + computer assisted surgery : MRCAS. 2011;7:452-8.

[18] Kim MC, Heo GU, Jung GJ. Robotic gastrectomy for gastric cancer: surgical techniques and clinical merits. Surgical endoscopy. 2010;24:610-5.

[19] Junfeng Z, Yan S, Bo T, Yingxue H, Dongzhu Z, Yongliang Z, et al. Robotic gastrectomy versus laparoscopic gastrectomy for gastric cancer: comparison of surgical performance and short-term outcomes. Surgical endoscopy. 2014;28:1779-87.

[20] Kang BH, Xuan Y, Hur H, Ahn CW, Cho YK, Han SU. Comparison of Surgical Outcomes between Robotic and Laparoscopic Gastrectomy for Gastric Cancer: The Learning Curve of Robotic Surgery. Journal of gastric cancer. 2012;12:156-63.

[21] Eom BW, Yoon HM, Ryu KW, Lee JH, Cho SJ, Lee JY, et al. Comparison of surgical performance and short-term clinical outcomes between laparoscopic and robotic surgery in distal gastric cancer. European journal of surgical oncology : the journal of the European Society of Surgical Oncology and the British Association of Surgical Oncology. 2012;38:57-63.

[22] Son T, Lee JH, Kim YM, Kim HI, Noh SH, Hyung WJ. Robotic spleen-preserving total gastrectomy for gastric cancer: comparison with conventional laparoscopic procedure. Surgical endoscopy. 2014;28:2606-15.

[23] Kim HH, Hyung WJ, Cho GS, Kim MC, Han SU, Kim W, et al. Morbidity and mortality of laparoscopic gastrectomy versus open gastrectomy for gastric cancer: an interim report--a phase III multicenter, prospective, randomized Trial (KLASS Trial). Annals of surgery. 2010;251:417-20.

[24] Hyun MH, Lee CH, Kwon YJ, Cho SI, Jang YJ, Kim DH, et al. Robot versus laparoscopic gastrectomy for cancer by an experienced surgeon: comparisons of surgery, complications, and surgical stress. Annals of surgical oncology. 2013;20:1258-65.

[25] Park JY, Jo MJ, Nam BH, Kim Y, Eom BW, Yoon HM, et al. Surgical stress after robot-assisted distal gastrectomy and its economic implications. The British journal of surgery. 2012;99:1554-61.

[26] Desiderio J, Stewart CL, Sun V, Melstrom L, Warner S, Lee B, et al. Enhanced Recovery after Surgery for Gastric Cancer Patients Improves Clinical Outcomes at a US Cancer Center. J Gastric Cancer. 2018;18:230-41.

[27] Tu RH, Lin JX, Desiderio J, Li P, Xie JW, Wang JB, et al. Does Intra-Abdominal Infection after Curative Gastrectomy Affect Patients' Long-Term Prognosis? A Multi-Center Study Based on a Large Sample Size. Surg Infect (Larchmt). 2019;20:271-7.

[28] Wu Z, Wang Q, Shi J, Cherry K, Desiderio J, Li Z, et al. [Diagnostic criteria and risk assessment of complications after gastric cancer surgery in western countries]. Zhonghua Wei Chang Wai Ke Za Zhi. 2017;20:135-9.

[29] Woo Y, Hyung WJ, Pak KH, Inaba K, Obama K, Choi SH, et al. Robotic gastrectomy as an oncologically sound alternative to laparoscopic resections for the treatment of early-stage gastric cancers. Archives of surgery. 2011;146:1086-92.

[30] Hiki N, Shimizu N, Yamaguchi H, Imamura K, Kami K, Kubota $\mathrm{K}$, et al. Manipulation of the small intestine as a cause of the increased inflammatory response after open compared with laparoscopic surgery. The British journal of surgery. 2006;93:195-204.

[31] Parisi A, Ricci F, Trastulli S, Cirocchi R, Gemini A, Grassi V, et al. Robotic Total Gastrectomy With Intracorporeal Robot-Sewn Anastomosis: A Novel Approach Adopting the Double-Loop Reconstruction Method. Medicine (Baltimore). 2015;94:e1922.

[32] Parisi A, Desiderio J. Establishing a multi-institutional registry to compare the outcomes of robotic, laparoscopic, and open surgery for gastric cancer. Surgery. 2015.

[33] Parisi A, Nguyen NT, Reim D, Zhang S, Jiang ZW, Brower ST, et al. Current status of minimally invasive surgery for gastric cancer: A literature review to highlight studies limits. International journal of surgery. 2015;17:34-40.

[34] Parisi A, Reim D, Borghi F, Nguyen NT, Qi F, Coratti A, et al. Minimally invasive surgery for gastric cancer: A comparison between robotic, laparoscopic and open surgery. World J Gastroenterol. 2017;23:2376-84.

[35] Clavien PA, Barkun J, de Oliveira ML, Vauthey JN, Dindo D, Schulick RD, et al. The Clavien-Dindo classification of surgical complications: five-year experience. Annals of surgery. 2009;250:18796. 